\title{
A EFETIVIDADE DO PADRÃO VENTILATÓRIO E INSPIRÔMETRO INCENTIVADOR EM PÓS OPERATÓRIO DE LAPAROTOMIA EM PACIENTES ONCOLÓGICOS
}

Débora Mayumi de Oliveira Kawakami, Guilherme Henrique Dalaqua Grande, Ana Maria Carrilho, Gabriela Ribeiro Menosse, Susimary Aparecida Trevisan Padulla, Rafael Zambeli Pinto

Universidade Estadual Paulista - UNESP, Departamento de Fisioterapia, Especialização/Residência em Fisioterapia Hospitalar, Presidente Prudente, SP. e-mail: debora_mayumi@hotmail.com

Agência de Fomento: CNPq-PIBIC

\section{RESUMO}

Em casos de câncer abdominal, uma das intervenções que podem ser realizadas é a laparotomia, no entanto, por ser próxima ao pulmão e muitas vezes seccionar músculos responsáveis pela respiração, causa alterações no padrão respiratório. O objetivo deste estudo foi analisar qual das intervenções fisioterápicas (inspirômetro de incentivo e padrão ventilatório) obteve o melhor desfecho associada à mobilização. Os pacientes foram separados em: G1 (exercícios de mobilização), G2 (exercícios de mobilização e inspirômetro incentivador) e G3 (exercícios de mobilização e padrão ventilatório). Embora existam estudos relacionados as técnicas de fisioterapia respiratória em casos de cirurgia abdominal, nenhum deles associou técnicas respiratórias com a mobilização precoce que é de grande importância para prevenção de complicações circulatórias, da mesma forma, nenhum estudo teve relação com pacientes oncológicos, que apresentam debilidade sistêmica. Foram avaliadas manovacuometria, EVA, HAD e mobilidade torácica, com inicio no 10 dia pós-operatório até o 5 o dia pós-operatório, com uma avaliação intermediária no 3 o dia. Foram encontradas diferenças estatísticas somente na análise intragrupos. São necessários mais estudos voltados para pacientes oncológicos.

Palavras-chave: Inspirômetro incentivador, padrão ventilatório, câncer, laparotomia, fisioterapia respiratória.

\section{EFFECTIVENESS OF A STANDARD VENTILATION AND INCENTIVE SPIROMETER IN POST SURGERY LAPAROTOMY IN PATIENTS ONCOLOGICAL}

\begin{abstract}
In abdominal cancer cases, one of the interventions que may be and a laparotomy, but not by be next the lung and many times sectioning muscles responsible for breathing causes no respiratory pattern changes. The aim of this study was to analyze which of physiotherapy interventions (spirometer incentive and ventilatory pattern) achieved the best outcome associated mobilization. The patients were separated into: G1 (mobilization exercises), G2 (mobilization exercises and spirometer incentive) and G3 (exercises of mobilization and ventilatory pattern). Although there related studies as respiratory physiotherapy techniques in abdominal surgery cases, none of them associated technical respiratory with early mobilization what is of great importance paragraph circulatory complications prevention, likewise no study had relations with cancer patients, which show weakness systemic. Were evaluated manovacuometry, EVA, ADH and thoracic mobility, starting in $1^{\text {st }}$ post-surgery until the $5^{\text {th }}$ post-surgery with an interim evaluation no $3^{\text {rd }}$ day. Were found differences in intragroup only statistics analysis. Further studies are needed directed to cancer patients.
\end{abstract}

Keywords: spirometer encourager, ventilatory pattern, cancer, laparotomy, respiratory therapy. 


\section{INTRODUÇÃO}

Com o passar dos anos a incidência do câncer vem aumentando e se tornou um problema de saúde pública mundial. Assim, estimativas para o ano de 2016/2017, no Brasil, haverá ocorrência de aproximadamente 597 mil novos casos de câncer, acometendo principalmente órgãos da região abdominal alta e baixa ${ }^{1}$.

Pacientes acometidos nessas regiões passam por procedimentos cirúrgicos com fins diagnóstico e terapêutico como, por exemplo, a laparotomia (abertura cirúrgica da cavidade abdominal), sendo indicada para se obter acesso a órgãos internos, para drenagem de líquidos e também para diagnóstico ${ }^{2}$.

A proximidade entre a cavidade abdominal, diafragma e pulmões aumenta o risco de problemas pulmonares. O local da incisão, os órgãos afetados, a inflamação pósoperatória e a dor no local da cirurgia podem comprometer a função pulmonar normal, afetando as capacidades e volumes pulmonares com redução de até $50 \%$ da capacidade vital e $70 \%$ da capacidade residual funcional ${ }^{3}$.

Dentre as estratégias a serem utilizadas para minimizar as complicações pulmonares pós-cirúrgicas, está a fisioterapia respiratória que utiliza algumas técnicas de reexpansão pulmonar como o padrão ventilatório e o inspirômetro fluxométrico Respiron ${ }^{\circledR 4}$.

Outra abordagem fisioterapêutica importante é a mobilização precoce, no qual foi evidenciado melhora na funcionalidade pela aceleração do retorno as atividades de vida diária, redução da dispneia e fadiga, tempo de internação e consequentemente custos hospitalares ${ }^{5}$.

A partir disso, ao observar um paciente internado com praticamente todos os comprometimentos já descritos, é possível estabelecer uma relação entre o câncer, o aspecto físico e emocional do paciente, provocando conflitos e desequilíbrio entre corpo e mente. Dessa forma, a ansiedade, depressão e dor são fatores que contribuem para estabelecer parâmetros sobre o estado físico e emocional de cada paciente ${ }^{6}$.

Mesmo havendo diversos trabalhos relacionados às técnicas fisioterapêuticas de padrão ventilatório e inspirômetro incentivador, nenhum estudo associou ambas as técnicas à mobilização precoce que é de grande importância para a prevenção de complicações circulatórias como a trombose venosa profunda e ao edema devido ao imobilismo ${ }^{7}$.

O objetivo do presente estudo foi comparar a eficácia de três intervenções fisioterapêuticas na expansibilidade torácica, intensidade da dor, níveis de ansiedade e depressão e força da musculatura respiratória em indivíduos oncológicos submetidos a laparotomia.

\section{METODOLOGIA}

Este ensaio clínico aleatorizado aconteceu na Santa Casa de Misericórdia de Presidente Prudente, SP. Foram incluídos pacientes com diagnóstico de câncer na região abdominal e pélvica, no primeiro dia de pós-operatório de laparotomia, e que tenham sido encaminhados para fisioterapia, obtendo ou não doenças associadas desde que não impossibilitassem a realização dos testes. Tendo como critérios de exclusão: instabilidade clínica, dispnéia em repouso, traqueostomia, Glasgow abaixo de 15, pacientes com antecedentes de pneumotórax espontâneo, aqueles que receberam alta antes do 3o dia de pós operatório e alterações cognitivas ou físicas (uso de prótese dentária) que impossibilitassem a realização dos testes. Esta pesquisa foi aprovada pelo comitê de ética CAAE: 50604315.9.0000.5402 e Parecer Núm.: 1.332.970.

Os participantes foram divididos aleatoriamente em Grupo 1 composto por pacientes que realizaram mobilização precoce, Grupo 2 com pacientes que fizeram uso do inspirômetro incentivador (fluxométrico Respiron ${ }^{\circledR}$ ) associado a mobilização precoce, e Grupo 3 por pacientes 
que realizaram técnica de padrão ventilatório associada a mobilização precoce.

Para realização das técnicas de fisioterapia respiratória e mobilização precoce os pacientes foram orientados a permanecer no leito em Fowler $45^{\circ}$.

A mobilização precoce, foi realizada a partir de manobras de mobilização ativa ou ativa-assistida de acordo com o grau de independência do paciente. Cada manobra foi executada em uma série de 5 repetições para cada articulação ${ }^{8}$.

A utilização do aparelho inspirômetro incentivador fluxométrico Respiron ${ }^{\circledR}$ do tipo Classic, foi realizada durante 3 séries de 10 repetições, com pausa de um minuto entre cada série. Os pacientes foram orientados a utilizarem o inspirômetro incentivador na vertical e realizar inspirações lentas, máximas e sustentadas de modo que houvesse elevação sequencial das três esferas do aparelho, mantendo-as elevadas por 3 segundos, com pausa de um minuto entre cada série ${ }^{9}$.

No grupo que realizou padrão ventilatório, os pacientes foram instruídos a realizar uma inspiração máxima com apneia sustentada e expirar lenta e suavemente com freno labial. Em seguida realizou a inspiração fracionada, e expiração em freno labial. Cada manobra foi executada pelo paciente em 2 séries com 10 repetições ${ }^{10}$.

Cada grupo realizou as intervenções duas vezes ao dia por cinco dias consecutivos e as avaliações ocorreram no 1 으, 3 으 e 50 dia de pós operatório, sendo composta por uma ficha de avaliação, mensuração da manovacuometria através da PIMáx (pressão inspiratória máxima) e PEMáx (pressão expiratória máxima), mobilidade torácica (com o auxílio de uma fita métrica, o tórax foi medido estando o paciente na fase final da expiração e em seguida pediu-se uma inspiração profunda e mediu-se novamente o diâmetro do tórax. Foram realizadas 3 medidas, sendo elas: mamilar, xifóide e umbilical, intensidade da dor (Escala visual analógica de dor-EVA: contando com as expressões faciais extremas que vão desde uma expressão sorridente de nenhuma dor (0) até dor intensa (10) com expressão triste e angustiada, sendo que os pacientes avaliaram sua dor diariamente) e o nível de ansiedade e depressão (Escala Hospitalar de Ansiedade e Depressão: composto por 14 perguntas, sendo sete voltadas para mensurar a depressão (HADS-D) e sete para mensurar a ansiedade (HADS-A), sendo uma pontuação entre 8 e 10 indicam um caso leve, 11-14 um caso moderado, e 15 ou acima, um caso grave de ansiedade e depressão).

A análise dos dados foi realizada pelo programa SPSS 17.0 atribuindo-se o nível de significância de $p<0,05$. Inicialmente foi realizado uma estatística descritiva para caracterização da amostra. Para a comparação intra-grupo (avaliação préintervenção versus avaliação pósintervenção) e assumindo uma distribuição normal para os desfechos, o teste $T$ pareado foi utilizado para a comparação entre os desfechos mensurados na avaliação inicial e final. Para a comparação entre-grupos e assumindo distribuição normal para os desfechos, a ANOVA foi para utilizado para a comparação entre grupos das médias mensuradas na avaliação pós-intervenção.

\section{RESULTADOS}

Com os dados obtidos podemos observar que na análise entre grupos, nenhum dos desfechos estudados (manovacuometria, HADS, EVA e cirtometria torácica) obtiveram resultados estatisticamente significativos, contrastando com os resultados obtidos na análise intragrupo, no qual os resultados de depressão e cirtometria (padrão ventilatório + mobilização precoce) na região mamilar e umbilical (mobilização precoce e padrão ventilatório + mobilização precoce) foram estatisticamente significativos.

De Agosto de 2014 à Setembro de 2015, apenas 19 pacientes foram recrutados e alocados nos grupos G1 (n=6), G2 (n=6) e G3 $(n=7)$. Durante a pesquisa, dois pacientes pertencentes ao G2 faleceram e uma 
paciente previamente alocada também no G2 foi excluída do estudo devido à não compreensão para realizar os testes da avaliação (principalmente para mensurar a mobilidade torácica). Do total de 19 pacientes recrutados, $8(42,1 \%)$ eram do sexo masculino e 11 (57,9\%) eram do sexo feminino. A idade média $\pm D P$, para o $G 1$ foi de $59,1 \pm 15,5$ anos, para o $G 2$ foi de $58 \pm 12,5$ anos e para o G3 foi de $59,1 \pm 15,5$ anos (Tabela 1).

Tabela 1: Caracteristicas da amostra, por grupos de intervenção.

\begin{tabular}{|c|c|c|c|}
\hline & $\begin{array}{c}\text { Grupo } 1 \\
\text { Mobilização }(n=6) \\
\text { i }\end{array}$ & $\begin{array}{l}\text { Grupo } 2 \\
\text { Inspirômetro } \\
\text { incentivador }(n=6)\end{array}$ & $\begin{array}{l}\text { Grupo } 3 \\
\text { Padrăo } \\
\text { ventilatório } \\
(n=7)\end{array}$ \\
\hline $\begin{array}{l}\text { Idade - media (DP) } \\
\text { Sexo } n(\%)\end{array}$ & $\begin{array}{r}59.1 \\
(15.5)\end{array}$ & $58.0(12.5)$ & $49.5(13.6)$ \\
\hline Feminino & $5(45.5 \%)$ & $4(36.4 \%)$ & $2(18.2 \%)$ \\
\hline & \multicolumn{2}{|c|}{ Tabagista $n(\%)$} & $5(62.5 \%)$ \\
\hline $\begin{array}{l}\text { Ex-fumante } \\
\text { Fumante } \\
\text { N-Fumante } \\
\text { - tolusta n( } 6)\end{array}$ & $\begin{array}{l}2(40.0 \%) \\
0(0.0 \%) \\
4(30.8 \%)\end{array}$ & $\begin{array}{l}0(0.0 \%) \\
0(0.0 \%) \\
6(46.2 \%)\end{array}$ & $\begin{array}{l}3(60.0 \%) \\
1(100.0 \%) \\
3(23.1 \%)\end{array}$ \\
\hline $\begin{array}{l}\text { Etulısta } n(\%) \\
\text { Năo } \\
\text { Sım }\end{array}$ & $\begin{array}{l}6(35.3 \%) \\
0(0.0 \%)\end{array}$ & $\begin{array}{l}6(35.3 \%) \\
0(0.0 \%)\end{array}$ & $\begin{array}{c}5(29.4 \%) \\
2(100.0 \%)\end{array}$ \\
\hline \multicolumn{4}{|l|}{ Hipertensão n(\%) } \\
\hline $\begin{array}{l}\text { Nao } \\
\text { Sim }\end{array}$ & $\begin{array}{l}4(33.3 \%) \\
2(28.6 \%)\end{array}$ & $\begin{array}{l}3(25.0 \%) \\
3(42.9 \%)\end{array}$ & $\begin{array}{l}5(41.7 \%) \\
2(28.6 \%)\end{array}$ \\
\hline \multicolumn{4}{|l|}{ Diabetes $n(\%)$} \\
\hline Nao & $\begin{array}{c}2(18.2 \%) \\
4(50 \%)\end{array}$ & $\begin{array}{l}3(27.3 \%) \\
3(37.5 \%)\end{array}$ & $\begin{array}{l}6(54.5 \%) \\
1(12.5 \%)\end{array}$ \\
\hline \multicolumn{4}{|l|}{ Colesterol n(\%) } \\
\hline $\begin{array}{l}\text { Sim } \\
\text { Nảo } \\
\text { Sedentarismo n(\%) }\end{array}$ & $\begin{array}{l}2(40.0 \%) \\
3(23.1 \%)\end{array}$ & $\begin{array}{l}3(60.0 \%) \\
3(23.1 \%)\end{array}$ & $\begin{array}{c}0(0.0 \%) \\
7(53.8 \%)\end{array}$ \\
\hline Sim & $\begin{array}{l}3(30.0 \%) \\
3(33.3 \%)\end{array}$ & $\begin{array}{l}4(40.0 \%) \\
2(22.2 \%)\end{array}$ & $\begin{array}{l}3(30.0 \%) \\
4(44.4 \%)\end{array}$ \\
\hline \multicolumn{4}{|c|}{$\begin{array}{l}\text { Histórico familiar de CA } \\
\mathrm{n}(\%)\end{array}$} \\
\hline $\begin{array}{l}\text { Sim - parente distante } \\
\text { Sim-parente proximo } \\
\text { Nao }\end{array}$ & $\begin{array}{l}0(0.0 \%) \\
4(33.3 \%) \\
2(33.3 \%)\end{array}$ & $\begin{array}{l}0(0.0 \%) \\
5(41.7 \%) \\
1(16.7 \%)\end{array}$ & $\begin{array}{c}1(100.0 \%) \\
3(25.0 \%) \\
3(50 \%)\end{array}$ \\
\hline
\end{tabular}

A partir dos dados coletados por meio da Escala Hospitalar de Ansiedade e Depressão, pode-se observar os resultados na Tabela 2 para os níveis de ansiedade e depressão pré e pós intervenção fisioterápica (intra grupos).

Tabela 2: Análise estatistica dos niveis de ansiedade e depressão, coletados a partir da aplicação do Questionário Hospitalar de Ansiedade e Depressão.

\begin{tabular}{|c|c|c|c|}
\hline & $\begin{array}{c}\text { Grupo } 1 \\
\text { Mobilizaçäo }(n=6)\end{array}$ & $\begin{array}{c}\text { Grupo } 2 \\
\text { Inspirômetro } \\
\text { Incentivador } \\
(n=6)\end{array}$ & $\begin{array}{c}\text { Grupo } 3 \\
\text { Padräo } \\
\text { Ventilatório } \\
(n=7)\end{array}$ \\
\hline \multicolumn{4}{|c|}{ Ansiedade - media (DP) } \\
\hline $1^{\circ} \mathrm{P} . \mathrm{O}$ & $9.0(5.4)$ & $6.6(2.0)$ & $6.5(4.6)$ \\
\hline Final & $7.6(5.2)$ & $5.2(2.8)$ & $2.8(2.7)$ \\
\hline \multicolumn{4}{|c|}{ Depressão - media (DP) } \\
\hline $1^{\circ} \mathrm{P} . \mathrm{O}$ & $2.6(3.8)$ & $2.3(2.3)$ & $3.4(2.5)$ \\
\hline Final & $4.4(5.3)$ & $1.7(0.5)$ & $1.2(3.4)^{*}$ \\
\hline
\end{tabular}

Dados de média (desvio padrão). 1ํ P.O. - primeiro dia de pós-operatório ou de intervenção fisioterápica; Final, último dia de intervenção fisioterápica. * ${ }^{*}<0,05$ para comparação intragrupos. 
Para avaliação da mobilidade torácica, foram realizadas três medidas para cada região (mamilar, xifoide e umbilical) sendo tabulada a maior dentre as três. Os dados estão representados na Tabela 3 para os três grupos de intervenção, com medidas no 10 dia de pós operatório e ao final das intervenções de cada grupo.

\begin{tabular}{|c|c|c|c|}
\hline & $\begin{array}{c}\text { Grupo } 1 \\
\text { Mobilização }(n=6)\end{array}$ & $\begin{array}{c}\text { Grupo } 2 \\
\text { Inspirômetro } \\
\text { Incentivador } \\
(n=6)\end{array}$ & $\begin{array}{c}\text { Grupo } 3 \\
\text { Padräo } \\
\text { Ventilatório } \\
(n=7)\end{array}$ \\
\hline \multicolumn{4}{|c|}{ Mamilar - media (DP) } \\
\hline $1^{\circ}$ P.O. & $0.4(0.4)$ & $0.4(0.4)$ & $0.5(0.4)$ \\
\hline Final & $0.6(0.8)$ & $0.7(0.2)$ & $1.0(0.6)^{*}$ \\
\hline \multicolumn{4}{|c|}{ Xifóide - media (DP) } \\
\hline $1^{\circ}$ P.O. & $0.1(0.2)$ & $0.3(0.4)$ & $0.2(0.5)$ \\
\hline Final & $0.4(0.4)$ & $0.7(0.2)$ & $0.5(0.4)$ \\
\hline \multicolumn{4}{|c|}{ Umbilical - media (DP) } \\
\hline $1^{\circ} \mathrm{P} . \mathrm{O}$ & $0.2(0.2)$ & $0.5(0.5)$ & $0.2(0.2)$ \\
\hline Final & $1.2(0.4)^{*}$ & $2.2(0.9)$ & $1.6(0.8)^{*}$ \\
\hline
\end{tabular}

Dados de média (desvio padrão). 10 P.O. - primeiro dia de pós-operatório ou de intervenção fisioterápica; Final, último dia de intervenção fisioterápica. * $p<0,05$ para comparação intragrupos.

Para a análise da força muscular respiratória a partir da manovacuometria, foram considerados valores pré (1ㅇ dia de pós-operatório) e pós-intervenção fisioterápica (Final), assim como descritos na Tabela 4.

Tabela 4: Força muscular respiratória avaliada através da manovacuometria no $1^{\circ}$ dia de pós operatório e $3 \% 5^{\circ}$ dia de pós operatório.

\begin{tabular}{cccc}
\hline & $\begin{array}{c}\text { Grupo 1 } \\
\text { Mobilização (n=6) }\end{array}$ & $\begin{array}{c}\text { Grupo 2 } \\
\text { Inspirômetro } \\
\text { Incentivador } \\
(\mathbf{n}=6)\end{array}$ & $\begin{array}{c}\text { Grupo 3 } \\
\text { Padrão } \\
\text { Ventilatório (n=7) }\end{array}$ \\
\hline PIMÁX - média (DP) & $19.6(16.4)$ & $18.3(11.4)$ & $32.5(15.7)$ \\
$1^{\circ}$ P.O. & $24.8(20.2)$ & $27.0(15.1)$ & $42.2(19.1)$ \\
Final & $10.6(6.5)$ & $26.6(27.3)$ & $35.7(20.7)$ \\
PEMÁX - média (DP) & $14.8(10.3)$ & $32.0(22.3)$ & $40.0(20.0)$ \\
\hline $1^{\circ}$ P.O. & &
\end{tabular}

Dados de média (desvio padrão). PIMÁX - pressão inspiratória máxima; PEMÁX - pressão expiratória máxima; 10 P.O. primeiro dia de pós-operatório ou de intervenção fisioterápica; Final, último dia de intervenção fisioterápica.

Em relação a dor, em todos os grupos é possível observar diminuição do nível de dor relatado pelo paciente após a intervenção fisioterápica analisada a partir da Tabela 5.

Tabela 5 Avaliação da dor, a partir da Escala Visual Analógica da Dor.

\begin{tabular}{|c|c|c|c|}
\hline & $\begin{array}{c}\text { Grupo } 1 \\
\text { Mobilização }(n=6)\end{array}$ & $\begin{array}{c}\text { Grupo } 2 \\
\text { Inspirômetro } \\
\text { Incentivador } \\
(\mathrm{n}=6)\end{array}$ & $\begin{array}{c}\text { Grupo } 3 \\
\text { Padrão ventilatório } \\
(\mathrm{n}=7)\end{array}$ \\
\hline $1^{\circ}$ P.O. & $3.5(3.0)$ & $1.6(1.9)$ & $1.1(1.5)$ \\
\hline Final & $0.8(1.7)$ & $0.7(1.5)$ & $0.2(0.4)$ \\
\hline
\end{tabular}

Dados de média (desvio padrão). 10 P.O. - primeiro dia de pós-operatório ou de intervenção fisioterápica; Final, último dia de intervenção fisioterápica. 


\section{DISCUSSÃO}

Devido ao pequeno tamanho amostral e, consequentemente, a falta de poder estatístico para rodar uma análise mais robusta, os resultados do presente estudo devem ser interpretados com ponderação. De acordo com os resultados, nenhum dos desfechos avaliados obtiveram significância estatística quando analisados entre os grupos, no entanto na análise intra grupos pode-se observar alguns desfechos significantes estatisticamente.

Pelo nosso conhecimento esse é o primeiro estudo a avaliar tais técnicas na população oncológica. Apesar de ambas as técnicas serem muito estudadas em relação a outras populações, faz-se necessário estudos voltados aos aspectos da oncologia, por se tratar de uma doença que proporciona debilidade sistêmica principalmente devido aos tratamentos utilizados.

Sendo assim, em relação à mobilidade torácica quando comparada intragrupos pode-se observar melhora na mobilidade torácica no Grupo 1 (mobilização precoce) e no Grupo 3 (padrão ventilatório + mobilização precoce).

Em estudo realizado com pacientes críticos, pode-se observar que houve aumento na mobilidade torácica em pacientes que realizaram técnicas de mobilização, porém não há estudos publicados com a mesma população deste estudo ${ }^{11}$.

Em relação à ansiedade e depressão, pode-se observar que na análise intragrupos houve diminuição estatisticamente significativa dos níveis de depressão no Grupo 1 que realizou o padrão ventilatório associado à mobilização precoce.

Estudo, revela que adicionalmente ao uso de fármacos, pode se estabelecer benefícios quando associadas às terapias psicossociais sendo uma delas a técnica respiratórias de relaxamento, podendo, desta forma, estar relacionada com o padrão ventilatório utilizado, o que provavelmente favoreceu a melhora dos pacientes de $\mathrm{G} 1 \mathrm{em}$ relação à depressão ${ }^{12}$.
Em relação a força muscular respiratória, ambas as análises (intra-grupos e intergrupos) não obtiveram dados estatisticamente significativos. Contudo, não foram encontrados estudos com esse delineamento na literatura.

Também observamos estabilidade em relação à quantificação da dor desde o 10 dia de pós-operatório até 0 50 dia de pósoperatório, gerados pela utilização de analgésicos ${ }^{13}$. Sendo assim, estes dados também não obtiveram resultado estatisticamente significativos.

Corroborando com os achados deste estudo está a revisão sistemática ${ }^{14}$ e estudo clínico que não estabelecem pesquisas que comprovem a superioridade do inspirômetro incentivador em relação ao padrão ventilatório na prevenção de complicações pulmonares após cirurgias abdominais ${ }^{15}$. Tal fato ocorreu devido à diversidade nas metodologias aplicadas e tamanho amostral.

\section{CONCLUSÃO}

Diante do exposto, faz-se necessário mais estudos nesta área, uma vez que o inspirômetro de incentivo e os padrões ventilatórios assim como a mobilização são amplamente utilizados em âmbito hospitalar e não obteve-se o melhor desfecho entre as três técnicas fisioterapêuticas. Além do mais, atualmente, são poucos os estudos na literatura voltados especificamente a fisioterapia convencional em pacientes oncológicos.

\section{REFERÊNCIAS}

1. INCA, 2015. Instituto Nacional do Câncer. Estimativa 2015. Incidência de Câncer no Brasil. Rio de Janeiro (BR)

2. Souza RP. Caracterização do perfil de pacientes no período pós-operatório de laparotomia em clínica cirúrgica. 2013. 61 f. Monografia (Bacharelado em Enfermagem)-Universidade de Brasília, Ceilândia-DF, 2013.

3. Mello M, Tanaka C, Dulle FL. Effects of an exercise program on muscle performance in patients undergoing allogeneic bone marrow transplantation. Bone Marrow Transplant. 
2003;32(7):723-8.

DOI: https://doi.org/10.1038/sj.bmt.1704227

4. Murakami FM, Yamaguti WP, Onoue MA, Mendes JM, Pedrosa RS, Maida AL, Kondo, CS, Salles ICD, Brito MMC, Rodrigues KM. Functional evolution of critically ill patients undergoing an early rehabilitation protocol. Rev Bras Ter Intensiva. 2015;27(2):161-9. DOI: https://doi.org/10.5935/0103-507x.20150028

5. Souza FB, Pires HF, Dewulf SLN, Inocenti A, Silva CBEA, Miasso IA. Pacientes em uso de quimioterápicos: depressão e adesão ao tratamento. Rev Esc Enferm USP. 2012;47(1):618.

6. Zafiropoulos B, Alison JA, MacCarren B. Physiological responses to the early mobilization of the intubed, ventilated abdominal surgery patients. Aust J physiother. 2004;50:95-100. DOI: https://doi.org/10.1016/S0004-9514(14)60101-X 7. Borges MV, Oliveira CRL, Peixoto E, Carvalho AAN. Fisioterapia motora em pacientes adultos em terapia intensiva. Rev Bras Ter Intensiva. 2009;21(4):446-52. DOI: htto de incentivo com três diferentes interfaces em pacientes submetidos à cirurgias torácicas e abdominais altas. ASSOBRAFIR Ciência 2(1):39-47, 2011.

9. Renault JA, Costa-Val R, Rossetti MB. Fisioterapia respiratória na disfunção pulmonar pós-cirurgia cardíaca. Rev Bras Cir Cardiovasc 2008;23(4):562-9.

DOI:

https://doi.org/10.1590/s0102-

76382008000400018

10. Sibinelli $M$ et al. Efeito imediato do ortostatismo em pacientes internados na unidade de terapia intensiva de adultos. Rev Bras Ter Intensiva. 2012;1(24):64-70.

11. Bottino SMB, Fráguas $R$, Gattaz FW. Depressão e câncer. São Paulo. Rev Psiq Clín. 2009;36(3):109-15.

DOI:

https://doi.org/10.1590/S0101$\underline{60832009000900007}$

12. Silva FA, Lopes TM, Duarte J, Medeiros RF. Tratamento fisioterapêutico no pós-operatório de laparotomia. J Health Sci Inst. 2010;28(4):3414.

13. Nascimento Junior $P$, Módolo NSP, Andrade $S$, Guimarães MMF, Braz LG, El Dib R. Incentive spirometry for prevention of postoperative pulmonary complications in upper abdominal surgery (Review). The Cochrane Collaboration. Published by John Wiley \& Sons, Ltd. 2014.

14. Tyson AF, Kendig CE, Mabedi C, Cairns BA, Charlis AG. The effect of incentive spirometry on postoperative pulmonary function following laparotomy: a randomized clinical trial. JAMA Surgery. 2015;150(3):229-36. DOI: https://doi.org/10.1001/jamasurg.2014.1846

Recebido para publicação em 16/08/2016

Revisado em 17/08/2016

Aceito em 22/08/2016 